\title{
Método intensivo de terapia vocal para idosos
}

\author{
Intensive voice therapy for the elderly
}

\author{
Juliana Fernandes Godoy 1,2 (1), Kelly Cristina Alves Silverio ${ }^{2,3}$ (1) , Eduardo Carvalho de Andrade 4 (1), \\ Alcione Ghedini Brasolotto ${ }^{2,3}$ (1)
}

\section{RESUMO}

O objetivo deste relato de caso foi descrever os resultados da terapia fonoaudiológica intensiva, em um caso de presbifonia, e apresentar uma proposta de tratamento vocal para idosos, demonstrando seus resultados na qualidade vocal, qualidade de vida e imagem laríngea de um paciente com presbifonia. O programa Terapia Vocal para Idosos (TVI) é constituído por uma sequência de exercícios, que visam ao aumento da loudness, coordenação pneumofonoarticulatória, melhora na vibração de mucosa e equilíbrio de ressonância, além da variação e controle da frequência. O modelo terapêutico em questão foi aplicado em um indivíduo do gênero feminino, com 87 anos de idade e queixas de voz fraca e dificuldade em ser ouvido. As terapias ocorreram de maneira intensiva em 16 sessões com 30 minutos de duração, quatro vezes por semana e com realização de exercícios em casa. A experiência de aplicação do programa em formato intensivo mostrou efeitos positivos na qualidade vocal, com diminuição do desvio global da voz, soprosidade na emissão sustentada e rugosidade na emissão sustentada e fala. A melhora no desempenho vocal refletiu em aumento nos valores dos domínios físico e global do protocolo de qualidade de vida relacionada à voz. Houve, também, melhora no fechamento glótico. O tratamento vocal com o método TVI intensivo demonstra efeitos positivos no tratamento da presbifonia e tem o potencial de promover benefícios na qualidade vocal, na qualidade de vida relacionada à voz e nos aspectos laríngeos dos idosos.

Palavras-chave: Voz; Envelhecimento; Treinamento vocal; Disfonia; Distúrbios da voz

\begin{abstract}
The aim of this study is to describe the results of intensive voice therapy in a presbyphonia case and to propose a voice treatment program for the elderly by demonstrating vocal quality, quality of life and laryngeal image results. The Voice Therapy for the Elderly (VTE) program consisted of a sequence of exercises that aimed at increasing intensity, pneumo-articulatory coordination, mucosal vibration and resonance balance, and increasing frequency variation and control. The therapeutic model in question was applied to an 87-year-old female subject with complaints of a weak voice and difficulty to be heard by others. This therapy program was applied with an intensive approach over 16 sessions, 30 minutes each session, four times a week, with assigned homework. The intensive voice therapy format demonstrated positive effects on vocal quality with an overall reduction in voice deviation, breathiness, and roughness on sustained phonation and speech. Vocal performance improvement showed a subsequent increase in the physical and general aspects of voice-related quality of life. There was also improvement in glottic closure. Voice treatment via the VTE method, with an intensive approach revealed to be positive in the treatment of presbyphonia and can potentially promote benefits for the elderly in terms of voice quality, voice-related quality of life, and laryngeal changes.
\end{abstract}

Keywords: Voice; Aging; Vocal training; Dysphonia; Voice disorders

\footnotetext{
Trabalho realizado no Departamento de Fonoaudiologia, Faculdade de Odontologia de Bauru, Universidade de São Paulo - USP - Bauru (SP), Brasil. ${ }^{1}$ Departamento de Fonoaudiologia, Universidade Federal do Rio Grande do Norte - UFRN - Natal (RN), Brasil.

${ }^{2}$ Programa de Pós-graduação em Fonoaudiologia, Faculdade de Odontologia de Bauru, Universidade de São Paulo - USP - Bauru (SP), Brasil.

${ }^{3}$ Departamento de Fonoaudiologia, Faculdade de Odontologia de Bauru, Universidade de São Paulo - USP - Bauru (SP), Brasil.

${ }^{4}$ Faculdade de Odontologia de Bauru, Universidade de São Paulo - USP - Bauru (SP), Brasil.

Conflito de interesses: Não.

Contribuição dos autores: JFG concepção e desenho do estudo, coleta, análise e interpretação dos dados, escrita do manuscrito; KCAS revisão crítica do conteúdo intelectual do trabalho e aprovação da versão final do artigo; ECA coleta de dados e aprovação da versão final do artigo; AGB concepção e desenho do estudo, análise e interpretação dos dados, revisão crítica do conteúdo intelectual do trabalho e aprovação da versão final do artigo.
}

Financiamento: Este trabalho foi financiado pela Fundação de Amparo à Pesquisa do Estado de São Paulo - FAPESP, Brasil. Processo: 2013/09325-0.

Autor correspondente: Juliana Fernandes Godoy. E-mail: jugodoy@ufrn.edu.br

Recebido: Outubro 31, 2019; Aceito: Novembro 04, 2019 


\section{INTRODUÇÃO}

A terapia vocal deve ser a primeira escolha do tratamento para a presbifonia, de acordo com um estudo que analisou os resultados de ensaios clínicos randomizados, envolvendo abordagens cirúrgicas e terapia vocal. Os autores indicaram a terapia por ser menos invasiva e apresentar resultados positivos na qualidade de vida ${ }^{(1)}$. O estudo de Oates ${ }^{(2)}$ sobre terapia vocal em idosos mostrou diversidade entre as intervenções, bem como na metodologia de aplicação e avaliação dos modelos terapêuticos propostos. A maioria dos estudos utilizou métodos terapêuticos consagrados, como os Exercícios de Função Vocal $\left(\mathrm{EFV}^{(3)}\right.$ e o método Lee Silverman Voice Treatment $\left(\mathrm{LSVT}^{\circledR}\right)^{(4)}$. Entretanto, estes métodos não foram criados especificamente para serem aplicados em casos de presbifonia. O único tratamento desenvolvido especialmente para idosos é o método PhoRTE que aborda, primordialmente, os aspectos da fala em forte intensidade ${ }^{(5)}$.

Considerando os aspectos laríngeos e vocais afetados pelo envelhecimento, como atrofia das pregas vocais, fendas glóticas e alterações na onda mucosa ${ }^{(6)}$, que causam qualidade vocal soprosa, astênica e rugosa ${ }^{(7)}$, a literatura sugere que os objetivos e exercícios da terapia no idoso devam incluir aumento do suporte respiratório, adução glótica, fonação sustentada, estabilidade vocal, extensão vocal, exercícios para melhorar a ressonância e precisão articulatória ${ }^{(8)}$, além de promover aumento de energia fonatória ou esforço vocal ${ }^{(9)}$.

Desta forma, uma abordagem terapêutica com enfoque não somente na emissão vocal em forte intensidade, favorecendo, também, os aspectos de vibração de mucosa, ressonância, variação e controle da frequência e coordenação pneumofônica, pode trazer benefícios, auxiliando, até mesmo, em casos de início do processo de envelhecimento vocal. Além disso, a utilização de exercícios vocais variados, como é comum aos fonoaudiólogos brasileiros, pode favorecer o alcance de cada um os objetivos terapêuticos de maneira mais específica.

Com relação à frequência das sessões terapêuticas, não há definição do que seria o ideal. Entretanto, existem trabalhos enfatizando o benefício do tratamento intensivo ${ }^{(4)}$. Há a hipótese de que este tipo de abordagem favoreça o aprendizado motor, mudança de comportamento e melhor desempenho da musculatura, em tempo inferior ao da terapia tradicional.

Este relato de caso teve o objetivo de descrever os resultados da terapia fonoaudiológica intensiva e apresentar o programa Terapia Vocal para Idosos (TVI), um modelo de terapia vocal elaborado, especificamente, para tratar a presbifonia. Além disso, outro objetivo foi demonstrar os resultados nos aspectos vocais, na qualidade de vida relacionada à voz e na configuração laríngea de uma paciente idosa com presbifonia.

\section{APRESENTAÇÃO DO CASO CLÍNICO}

O modelo de terapia aqui proposto envolveu exercícios de diferentes técnicas, na intenção de trabalhar, em uma única sessão de terapia, mais de um objetivo terapêutico. Assim, ao longo do processo, o aprendizado do novo padrão de fonação tende a estabelecer-se de forma equilibrada. É importante acrescentar que o desenvolvimento do programa de terapia foi realizado por dois dos autores com experiência no tratamento vocal de idosos, após discutirem quais as técnicas comumente utilizadas no tratamento destes indivíduos. Depois de selecionada a sequência de exercícios, o programa foi aplicado em um estudo piloto, um ano antes de ser utilizado em pesquisas.

A terapia foi aplicada em 16 sessões, com duração de cerca de 30 minutos, quatro vezes por semana. A frequência e o número total das sessões teve como referência o método LSVT ${ }^{\circledR(4)}$ de terapia vocal, descrito como intensivo e com eficácia comprovada no tratamento do envelhecimento da voz. O indivíduo que participou deste estudo foi uma mulher com 87 anos de idade, apresentando queixas vocais relacionadas ao envelhecimento. Após avaliação fonoaudiológica e otorrinolaringológica inicial, obteve-se o diagnóstico de presbifonia.

Este estudo de caso provém de um estudo maior, aprovado pelo Comitê de Ética em Pesquisa da Faculdade de Odontologia de Bauru - Universidade de São Paulo (CAAE: 07814412.4.0000.5417, parecer: 160.793). A participante assinou o Termo de Consentimento Livre Esclarecido, concordando com os termos e objetivos do estudo.

Para a participação, foi necessário constatar que a paciente em questão apresentasse: condições gerais de saúde estável e condições cognitivas e motoras que permitissem a realização dos procedimentos de avaliação e terapia; queixas vocais originadas no período do envelhecimento; resposta positiva a, pelo menos, um exercício de cada uma das técnicas propostas no programa terapêutico; ausência de problemas neurológicos; ausência de alterações estruturais ou lesões laríngeas não compatíveis com as mudanças fisiológicas decorrentes da idade; ausência de histórico de cirurgia laríngea; nunca ter fumado; ausência de queixas ou dificuldades auditivas que comprometessem a compreensão das ordens dadas durante as avaliações e sessões de terapia.

Foi realizada gravação de uma emissão sustentada da vogal /a/ e de fala encadeada, com contagem de números até dez, na semana anterior ao início do tratamento e na semana após sua finalização. Para a gravação de voz, utilizou-se microfone de cabeça (AKG modelo C444PP), localizado a 45 graus e $4 \mathrm{~cm}$ de distância da boca da paciente. As gravações foram realizadas em uma sala acusticamente tratada, foi utilizado o software de áudio profissional Sound Forge Pro 10.0, placa de som Creative Audigy II, na taxa de $44.100 \mathrm{~Hz}$, canal Mono e 16 bits, com amplificador modelo 3710 (Pentax). O espectrograma da vogal sustentada foi extraído do programa Real-Time Spectrogram (Pentax), com filtro de banda estreita. A taxa de apresentação foi ajustada para $11.025 \mathrm{~Hz}, 16$ bits e resolução de $5.000 \mathrm{~Hz}$.

A participante também respondeu ao questionário de autoavaliação Qualidade de Vida em Voz (QVV), validado para o português brasileiro ${ }^{(10)}$, antes e após o tratamento. Além disso, foi realizada avaliação das estruturas laríngeas durante a fonação, por meio dos exames de telelaringoscopia, videoestroboscopia e nasolaringoscopia.

Todos os procedimentos de avaliação foram executados por um fonoaudiólogo e um otorrinolaringologista, sem nenhum acesso aos procedimentos do tratamento. Da mesma forma, as avaliações perceptuais da voz e da laringe foram feitas por avaliadores cegos, sem acesso ao tipo de tratamento realizado ou ao momento da avaliação (pré ou pós-tratamento).

Para a análise perceptivo-auditiva, um único fonoaudiólogo, com mais de cinco anos de experiência neste tipo de avaliação, recebeu um total de 126 pares de emissões da vogal sustentada /a/ e 126 pares de emissão da fala encadeada de indivíduos idosos com queixas vocais, que foram submetidos à terapia. Cada par correspondia a diferentes momentos de gravação de voz 
(antes ou após a terapia), porém, não havia identificação destes dados e os áudios eram apresentados de forma randomizada. Houve repetição de $10 \%$ da amostra para cálculo da confiabilidade interna do avaliador. Para análise, o avaliador deveria identificar se a segunda emissão apresentada melhorou, piorou ou não mostrou diferenças, quando comparada à primeira emissão. Tais comparações foram feitas em relação aos seguintes parâmetros: grau geral do desvio vocal, rugosidade, soprosidade, loudness e instabilidade (este último, apenas para a vogal sustentada).

Da mesma maneira, foi feita a análise perceptivo-visual de 126 pares de exames de laringe por um examinador experiente, com repetição de $10 \%$ do total da amostra. Os exames foram apresentados aos pares, sem identificação do paciente e do momento em que foram realizados. Os seguintes parâmetros foram observados: arqueamento de pregas vocais, proeminência de processos vocais, fechamento glótico, constrição mediana ou anteroposterior do vestíbulo laríngeo e alteração na vibração de mucosa. $\mathrm{O}$ avaliador deveria indicar se a presença de tais aspectos aumentou, diminuiu ou não mostrou diferenças entre os exames. Tanto o avaliador das vozes, quanto das imagens laríngeas, obtiveram confiabilidade substancial (coeficiente Kappa 0,78 e 0,75, respectivamente).

A seleção dos exercícios foi feita com base em sua fisiologia, com a intenção de melhorar os aspectos vocais alterados pelo envelhecimento. Todos os exercícios foram realizados em cada uma das sessões de terapia, que tiveram duração média de 30 minutos. Cada exercício proposto foi realizado por quatro minutos, contados com auxílio de um cronômetro, à exceção do último exercício, realizado por dois minutos, em virtude do esforço necessário para produzi-lo. Os exercícios do programa de terapia proposto estão descritos na Tabela 1.
A orientação foi de que a paciente realizasse o programa de exercício vocal em casa, duas vezes ao dia, nos dias em que não participasse da terapia fonoaudiológica presencial, e uma vez ao dia, no dia em que realizasse a sessão terapêutica. Esta prática foi iniciada a partir da quarta sessão presencial, quando a paciente já conseguia realizar os exercícios com autonomia.

Para a análise dos resultados da terapia, foi realizado o cálculo dos três escores do QVV: físico, socioemocional e total. A comparação entre eles mostrou aumento dos valores dos escores físico e total após a terapia vocal. Inicialmente, o escore físico era de $91,7 \%$ e, após a terapia, passou para $95,8 \%$; o escore socioemocional, antes do tratamento, era de $100 \%$ e manteve-se da mesma forma após a terapia; já o escore total, passou de $95 \%$ antes do início da intervenção vocal para $97,5 \%$, após a finalização.

A qualidade vocal apresentou-se rugosa e astênica antes do tratamento. Ao final do processo terapêutico, foi descrita melhora na projeção vocal, com aumento da loudness e diminuição na soprosidade e instabilidade. As melhoras ocorreram tanto para a emissão da vogal sustentada, quanto para a fala encadeada, o que proporcionou satisfação da família e da paciente quanto à efetividade de comunicação. Tais mudanças podem ser observadas por meio da análise espectrográfica da emissão da vogal sustentada /a/ em pitch e loudness habituais, no período pré-terapia (Figura 1) e no período após a intervenção (Figura 2).

Os achados laríngeos iniciais foram de arqueamento de pregas vocais, processos vocais salientes e fenda fusiforme anteroposterior à fonação (Figura 3). Ao final do processo terapêutico, a avaliação laríngea demonstrou melhora no fechamento glótico (Figura 4).

Tabela 1. Exercícios do programa de terapia vocal proposto

\begin{tabular}{ll}
\hline \multicolumn{1}{c}{ Técnica } & \multicolumn{1}{c}{ Exercício } \\
\hline Técnica de sons vibrantes & $\begin{array}{l}\text { Vibração de língua ou lábios, com emissão sonora } \\
\text { contínua. }\end{array}$ \\
Técnica dos sons nasais & $\begin{array}{l}\text { Emissão do som nasal "m", de forma contínua ou } \\
\text { "m" mastigado. }\end{array}$ \\
Técnica de escalas musicais & $\begin{array}{l}\text { Emissão vocal em escalas em glissando } \\
\text { ascendentes e descendentes, associados aos } \\
\end{array}$ \\
& sons facilitadores, sendo estes vibrantes, nasais ou \\
& fricativo "z".
\end{tabular}

Técnica de emissão em tempo Emissão de vogais sustentadas no tempo máximo máximo de fonação

de fonação, com intensidade vocal mais forte que o habitual, mantendo abertura de boca adequada, sem esforço muscular excessivo, controlando-se a qualidade vocal ao longo da emissão.

Fonação com tubo na água

Fonação com tubo profundo na água

\author{
Emissão prolongada de som semelhante a \\ "u" ou "v" em um tubo de silicone de $35 \mathrm{~cm}$ de \\ comprimento e $9-12 \mathrm{~mm}$ de diâmetro, colocado na \\ boca, entre ou em frente os dentes incisivos e acima \\ da língua, que deverá estar relaxada e poderá tocar \\ levemente o tubo, sem ocluí-lo, sendo que a outra \\ extremidade será mergulhada dentro de uma garrafa \\ pet de água, com capacidade para cerca de $500 \mathrm{ml}$ \\ de líquido e será preenchida com cerca de $2 / 3$ de \\ água. $\mathrm{O}$ tubo deverá ser imerso no líquido na \\ profundidade de 1-2 cm. \\ Emissão curta de som semelhante a "u" ou "v" em \\ um tubo de silicone, conforme descrito no exercício \\ anterior, porém, o tubo deverá ser imerso no \\ líquido na profundidade de $10 \mathrm{~cm}$.
}

Melhora a vibração de mucosa de pregas vocais e auxilia para uma produção vocal normotensa ${ }^{(11)}$.

Facilita a produção vocal com menor esforço, diminui a rugosidade e equilibra a ressonância ${ }^{(12)}$.

Aumenta a flexibilidade vocal ${ }^{(13)}$ e melhora o fechamento glótico ${ }^{(12)}$.

Aumenta os níveis de pressão sonora ${ }^{(14)}$, melhora a coaptação glótica e aumenta a resistência à passagem do ar expiratório, auxiliando na melhora da estabilidade fonatória. É de grande eficácia em situações de hipotonia vocal e, neste caso, o exercício deverá ser realizado em intensidade vocal levemente mais forte que a habitual|(12).

Amplia o trato vocal e reduz a força de colisão entre as pregas vocais ${ }^{(15)}$.

Ativa a musculatura laríngea e realiza compensação, como um exercício de esforço, auxiliando no fechamento glótico ${ }^{(15)}$. 


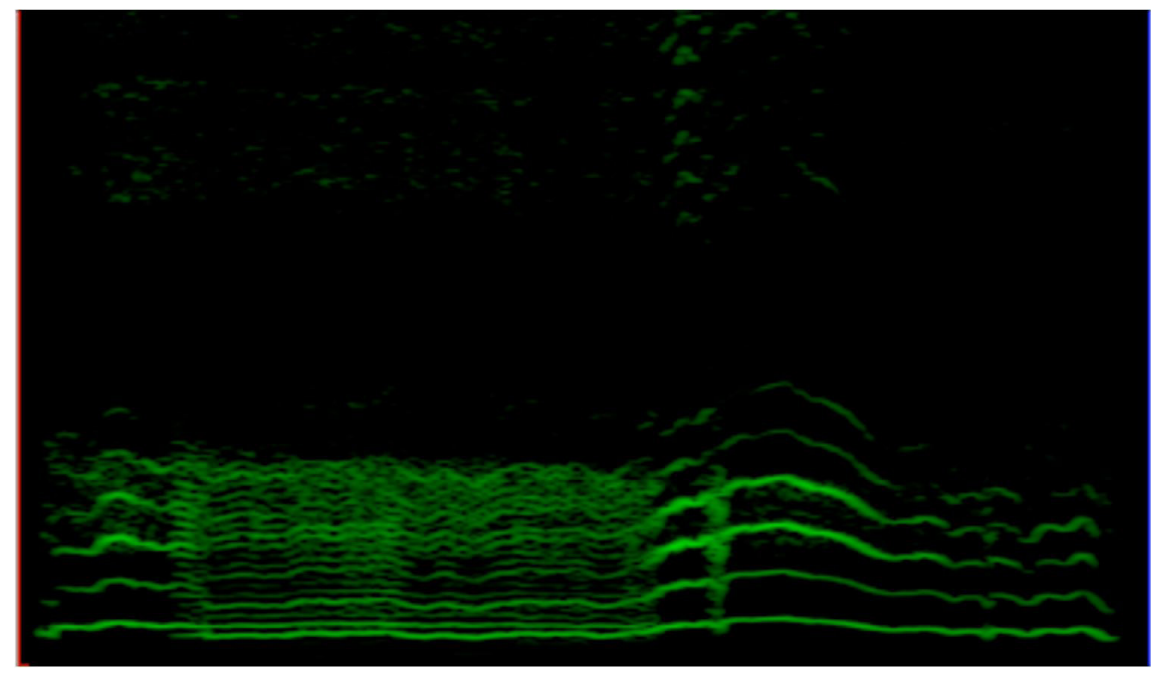

Figura 1. Imagem espectrográfica da emissão da vogal sustentada /a/ extraída antes do início da intervenção fonoaudiológica com o programa Real -Time Spectrogram (Pentax). Traçado irregular, com presença de ruído, poucos harmônicos e presença de sub-harmônicos, especialmente no início da emissão, além de instabilidade

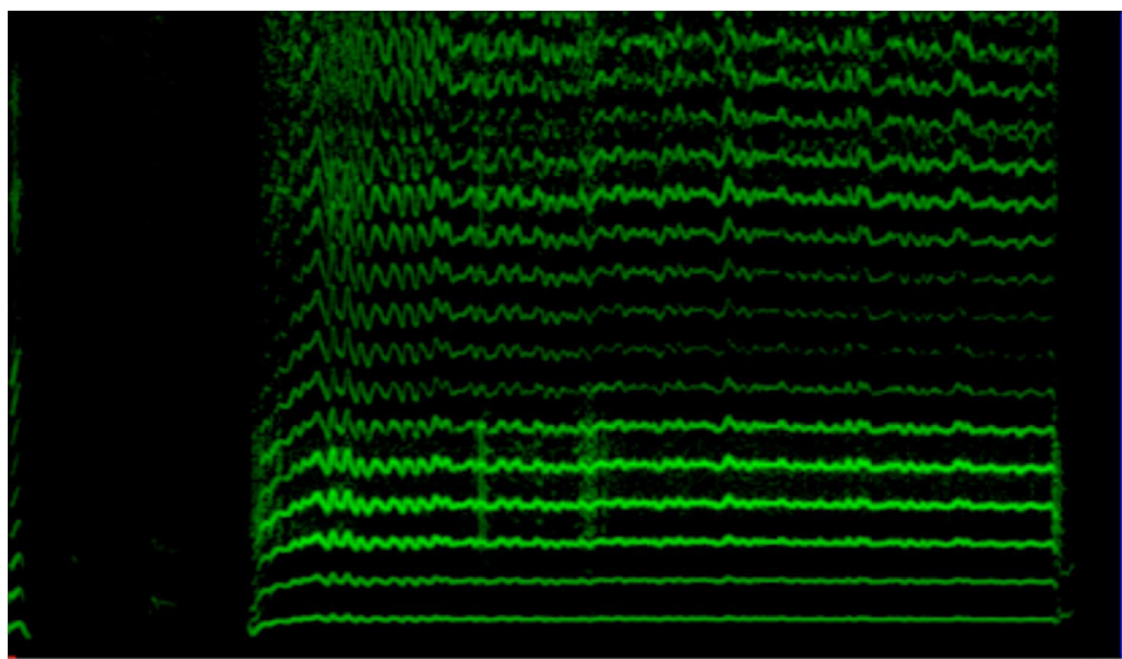

Figura 2. Imagem espectrográfica da emissão da vogal sustentada /a/ extraída após a finalização do processo terapêutico com o programa Real -Time Spectrogram (Pentax). Presença de maior número de harmônicos, ausência de sub-harmônicos, com redução de ruído e maior controle de frequência

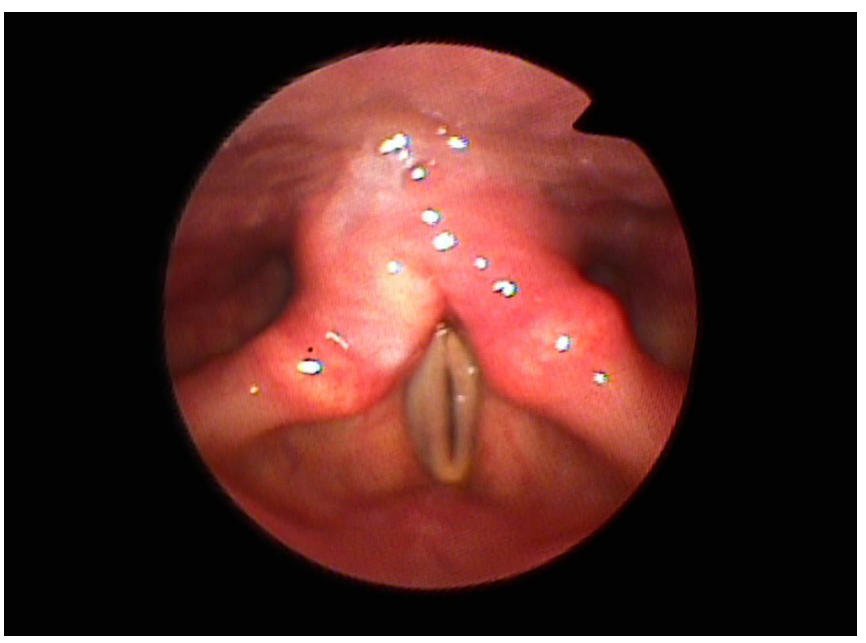

Figura 3. Laringoscopia realizada uma semana antes do início da terapia vocal

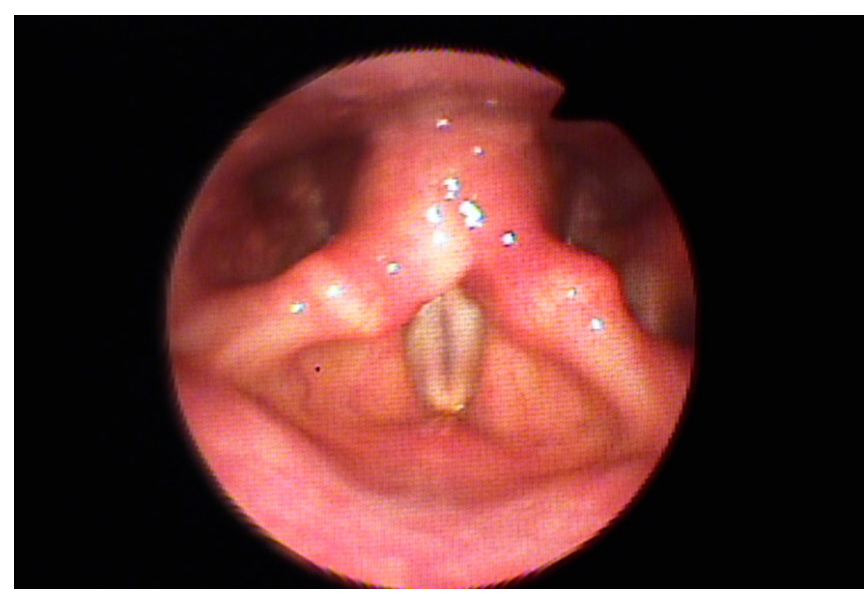

Figura 4. Laringoscopia realizada uma semana após a finalização do processo terapêutico 


\section{DISCUSSÃO}

$\mathrm{Na}$ fonoaudiologia brasileira, é comum a utilização combinada de exercícios vocais pertencentes a mais de um método terapêutico ${ }^{(12)}$, partindo do princípio de que a disfonia deriva de mais de um aspecto vocal alterado. Além disso, o uso de uma abordagem com diversificação dos exercícios permite ao clínico escolher exercícios de diversas técnicas e, até mesmo, misturá-los, o que pode tornar a terapia mais atrativa, permitindo alcançar vários objetivos terapêuticos.

O envelhecimento pode levar a modificações morfológicas da laringe ${ }^{(6)}$, podendo ocasionar alterações na vibração mucosa. Os exercícios vibrantes podem maximizar a amplitude de vibração da $\operatorname{mucosa}^{(13)}$ e permitir uma fonação mais confortável, com maior número de harmônicos, o que pode ter tido um papel importante neste caso, especificamente, conforme se observa no espectrograma após o tratamento (Figura 2).

Os exercícios nasais podem favorecer a ressonância mais equilibrada e auxiliar na melhoria da projeção vocal, reduzindo o uso excessivo da ressonância laríngea, o que pode ajudar a estabelecer um melhor equilíbrio durante a fonação e promover uma emissão com menor esforço, facilitando a produção vocal $^{(12)}$ e permitindo voz mais forte e projetada. Além disso, os pacientes com presbifonia tendem a compensar a voz fraca realizando esforço durante a fonação e os exercícios nasais parecem melhorar a loudness sem a realização de tensão adicional durante a emissão, o que pode auxiliar a performance vocal, até mesmo para a realização dos exercícios subsequentes.

Quanto às emissões com variações de frequência, os glissandos ascendentes e descendentes têm como objetivo trabalhar a extensão e flexibilidade vocal ${ }^{(13)}$. A literatura também discorreu sobre o fato desta técnica auxiliar no fechamento das fendas, ao produzir estiramento e encurtamento de pregas vocais ${ }^{(12)}$. Além disso, o método dos Exercícios de Função Vocal (EFV) também se utiliza desse tipo de exercício e tem demonstrado resultados positivos na aplicação em pacientes com presbifonia ${ }^{(3)}$.

Os exercícios que priorizam a emissão em tempo máximo de fonação ou a emissão sustentada em intensidade forte já são utilizados em terapias para presbifonia, uma vez que promovem maior estabilidade à fonação, melhor controle do fluxo aéreo e melhor projeção associada ao aumento da intensidade vocal. Tais resultados estão descritos na aplicação dos métodos $\mathrm{EFV}^{(3)}$, LSVT $^{\circledR(4)}$ e PhoRTE ${ }^{(5)}$, que fazem uso de exercícios similares.

Os exercícios de fonação em tubo mergulhado na água envolvem a oclusão parcial do trato vocal e são realizados com tubos ou canudos de diversos materiais e dimensões. A técnica do tubo de ressonância flexível, com material de silicone, é um destes métodos. Tais exercícios têm a intenção de promover a fonação mais equilibrada, com menor interferência das estruturas supraglóticas e há a possibilidade de utilização em uma configuração capaz de proporcionar melhora vocal nos casos de hipofonia. Para este ultimo objetivo, a literatura ${ }^{(15)}$ sugere a imersão mais profunda do tubo na água e a produção de emissões vocais curtas, gerando um esforço adicional que facilita a aproximação das pregas vocais e ativa o músculo tireoaritenóideo, levando a crer no potencial deste exercício em promover melhora no fechamento glótico.

Sugere-se, portanto, que a combinação dos exercícios utilizados tenha promovido a melhora na qualidade vocal e fechamento glótico da paciente em questão, considerando a literatura. Como o programa de terapia proposto é composto de exercícios que objetivam, entre outros aspectos, melhorar a vibração de mucosa, o fechamento glótico e aumentar a intensidade e estabilidade vocal, é esperada melhora nos parâmetros de rugosidade, soprosidade, bem como diminuição do desvio global da voz. Outros programas de terapia vocal aplicados em sujeitos com presbifonia, contendo um ou mais dos exercícios aqui propostos, também atingiram o objetivo de melhora nos parâmetros de qualidade vocal ${ }^{(2-4)}$. Tal melhora pôde ter influência direta nas questões relacionadas ao aspecto físico do questionário QVV, proporcionando melhora global nos resultados após a terapia vocal, ainda que a tal melhora tenha sido discreta, uma vez que a paciente já apresentava escores elevados de qualidade de vida em voz, antes da terapia. Outros estudos semelhantes também demonstraram resultados positivos nos questionários de autoavaliação em idosos submetidos à fonoterapia ${ }^{(2,5)}$.

Ressalta-se que alguns pacientes idosos com queixas vocais decorrentes do envelhecimento apresentam padrão de fala tenso, seja por comportamento vocal inadequado, ou como forma de compensação dos aspectos vocais alterados. Quando a tensão vocal for exacerbada, sugere-se a aplicação do TVI com modificações, ou associado a outros procedimentos terapêuticos, a fim de equilibrar a produção de voz mais forte (o que é um dos objetivos do programa), com tensão reduzida. O uso dos mesmos exercícios em cada sessão foi feito, aqui, com fins de pesquisa. Entretanto, o clínico deve utilizar as técnicas com as quais se sente confortável, desde que a fisiologia do exercício vocal esteja relacionada à fisiologia do mecanismo vocal do paciente.

Outro aspecto ao qual o fonoaudiólogo deve estar atento é a rouquidão molhada, presente, muitas vezes, por excesso de secreção acumulada na laringe desses pacientes. Nestes casos, é importante investigar aspectos digestivos alterados, especialmente quanto à presença de distúrbios da deglutição e refluxo laringofaríngeo, realizando as orientações e os encaminhamentos necessários.

Sugere-se, também, a realização dos exercícios associados à fala, para promover a generalização da melhora na qualidade vocal quando esta não é alcançada apenas com os exercícios do programa TVI. O caso apresentado melhorou a qualidade vocal, tanto na fonação sustentada, quanto na fala, porém, técnicas de fala com objetivo de promover a generalização também são importantes.

As pesquisas em tratamento dos distúrbios da voz são variáveis no que diz respeito ao número de sessões, frequência e duração da terapia fonoaudiológica. Tendo como base este fator, escolheu-se, para este estudo, a utilização do mesmo número de sessões terapêuticas do método vocal com maior comprovação científica e que é designado a pacientes com hipofonia, o LSVT ${ }^{\circledR(3)}$. Como o objetivo da reabilitação vocal na presbifonia é minimizar os efeitos da hipofunção laríngea, um programa de terapia intensiva enfatiza a repetição e estimula a melhor produção vocal possível, permitindo melhora e generalização mais rápidas.

Uma das limitações deste estudo está na descrição de um único caso e, por isso, é importante a aplicação do programa em uma amostra maior. Futuras pesquisas devem ser realizadas, investigando o tempo ótimo de realização de cada um dos exercícios vocais durante a terapia com o paciente idoso. Além disso, os ensaios clínicos controlados e randomizados produzirão evidências científicas quanto aos efeitos do TVI e do tratamento no formato intensivo. 


\section{COMENTÁRIOS FINAIS}

O TVI é um programa que tem se mostrado positivo no tratamento da presbifonia, especialmente pelo fato de ser voltado para as necessidades do idoso de forma abrangente e de envolver técnicas de conhecimento dos clínicos brasileiros. As técnicas utilizadas são de fácil acesso e aplicação e o programa demonstrou benefícios na qualidade de vida relacionada à voz e configuração laríngea do paciente submetido ao tratamento.

\section{REFERÊNCIAS}

1. Bradley JP, Hapner ER, Johns MM 3rd. What is the optimal treatment for presbyphonia? Laryngoscope. 2014;124(11):2439-40. http://dx.doi. org/10.1002/lary.24642. PMid:25132245.

2. Oates JM. Treatment of dysphonia in older people: the role of the speech therapist. Curr Opin Otolaryngol Head Neck Surg. 2014;22(6):477-86. http://dx.doi.org/10.1097/MOO.0000000000000109. PMid:25250623.

3. Kaneko M, Hirano S, Tateya I, Kishimoto Y, Hiwatashi N, Fujiu-Kurachi $\mathrm{M}$, et al. Multidimensional analysis on the effect of vocal function exercises on aged vocal fold atrophy. J Voice. 2015;29(5):1-7. http:// dx.doi.org/10.1016/j.jvoice.2014.10.017.

4. Lu F-L, Presley S, Lammers B. Efficacy of intensive phonatoryrespiratory treatment (LSVT) for presbyphonia: two case reports. J Voice. 2013;27(6):786.e11-23. http://dx.doi.org/10.1016/j.jvoice.2013.06.006.

5. Ziegler A, Verdolini Abbott K, Johns M, Klein A, Hapner ER. Preliminary data on two voice therapy interventions in the treatment of presbyphonia. Laryngoscope. 2014;124(8):1869-76. http://dx.doi. org/10.1002/lary.24548. PMid:24375313.

6. Yamauchi A, Yokonishi H, Imagawa H, Sakakibara K-I, Nito T, Tayama $\mathrm{N}$, et al. Quantification of vocal fold vibration in various laryngeal disorders using high-speed digital imaging. J Voice. 2015;29(6):75562. http://dx.doi.org/10.1016/j.jvoice.2014.12.008.
7. Menezes KSM, Master S, Guzman M, Bortnem C, Ramos LR. Differences in acoustic and perceptual parameters of the voice between elderly and young women at habitual and high intensity. Acta Otorrinolaringol Esp. 2014;65(2):76-84. http://dx.doi.org/10.1016/j. otoeng.2013.11.012. PMid:24439013.

8. Morrison M, Rammage L. The management of voice disorders. San Diego: Singular Publishing; 1994. Voice disorders in the elderly; p. 141-9. http://dx.doi.org/10.1007/978-1-4899-2903-7.

9. Gartner-Schmidt J, Rosen C. Treatment success for age-related vocal fold atrophy. Laryngoscope. 2011;121(3):585-9. http://dx.doi. org/10.1002/lary.21122. PMid:21344441.

10. Gasparini G, Behlau M. Quality of life: validation of the Brazilian version of the Voice-Related Quality of Life (V-RQOL) measure. J Voice. 2009;23(1):76-81. http://dx.doi.org/10.1016/j.jvoice.2007.04.005. PMid:17628396.

11. Zimmer V. Tempo ideal de vibração lingual sonorizada e qualidade vocal de mulheres [dissertação]. Santa Maria: Programa de Pósgraduação em Distúrbios da Comunicação Humana, Universidade Federal de Santa Maria; 2011.

12. Behlau M, Madazio G, Feijó D, Azevedo R, Gielow I, Rehder MI. Aperfeiçoamento vocal e tratamento fonoaudiológico das disfonias. In: Behlau M, editor. Voz: o livro do especialista. Rio de Janeiro: Revinter; 2005. p. 410-565.

13. Dargin TC, DeLaunay A, Searl J. Semioccluded vocal tract exercises: changes in laryngeal and pharyngeal activity during stroboscopy. J Voice. 2016 Maio;30(3):377.e1-9. http://dx.doi.org/10.1016/j. jvoice.2015.05.006. PMid:26390961.

14. Dromey C, Ramig LO, Johnson AB. Phonatory and articulatory changes associated with increased vocal intensity in Parkinson disease: a case study. J Speech Hear Res. 1995;38(4):751-64. http://dx.doi.org/10.1044/ jshr.3804.751. PMid:7474969.

15. Simberg S, Laine A. The resonance tube method in voice therapy: description and practical implementations. Logoped Phoniatr Vocol. 2007;32(4):165-70. http://dx.doi.org/10.1080/14015430701207790. PMid: 17852715. 